\title{
Successful Antemortem Diagnosis and Treatment of Pulmonary Tumor Thrombotic Microangiopathy
}

\author{
Kenjuro Higo ${ }^{1}$, Kayoko Kubota ${ }^{1}$, Ayano Takeda ${ }^{1}$, Michiyo Higashi ${ }^{2}$ and Mitsuru Ohishi ${ }^{1}$
}

\begin{abstract}
Pulmonary tumor thrombotic microangiopathy (PTTM) is a fatal cancer-related complication with rapid progression. Although the underlying molecular mechanisms of PTTM remain unclear, platelet-derived growth factor (PDGF) and vascular endothelial growth factor (VEGF) may play important roles in the pathogenesis of PTTM. We herein report a case of PTTM that was diagnosed antemortem by pathology using pulmonary wedge aspiration. The patient was treated with a combination therapy of imatinib (PDGF receptor antagonist), bevacizumab (VEGF receptor inhibitor), S-1, and cisplatin. These findings suggest that molecular-target drugs are effective for the treatment of PTTM.
\end{abstract}

Key words: pulmonary tumor thrombotic microangiopathy, pulmonary hypertension, bevacizumab, imatinib

(Intern Med 53: 2595-2599, 2014)

(DOI: 10.2169/internalmedicine.53.2379)

\section{Introduction}

Pulmonary tumor thrombotic microangiopathy (PTTM) is characterized by fibrocellular intimal proliferation of the small pulmonary arteries and arterioles in carcinoma patients (1). Von Herbay et al. first reported PTTM in 1990 where they noted that PTTM was observed in $3.3 \%$ of the autopsies of patients with malignant tumors (1). The diagnosis and treatment of PTTM in living patients is extremely difficult (2), and many patients die within several weeks due to right cardiac failure. We herein report a case of PTTM that was diagnosed and treated with a combination therapy of imatinib [platelet-derived growth factor (PDGF) receptor antagonist], bevacizumab [vascular endothelial growth factor (VEGF) receptor inhibitor], and chemotherapy with S-1 and cisplatin. The patient's symptoms dramatically improved following treatment.

\section{Case Report}

A 61-year-old man was diagnosed with colorectal cancer and had an abdominal operation in 2009. A pathological examination revealed tubular adenocarcinoma. The patient re- mained in good health for 16 months. However, recurrence of his colorectal cancer was detected in April 2011, and he underwent another operation. After surgery, chemotherapy with bevacizumab was administered.

From November 2012, the patient began to feel breathlessness and have a dry cough which gradually worsened. He visited a nearby hospital in December 2012 where transthoracic echocardiography detected pulmonary hypertension. Because adverse side effects of bevacizumab were suspected, the drug was discontinued and a diuretic was instead administered. However, his symptoms continued to worsen after bevacizumab discontinuation, and he was referred to our hospital in January 2013.

On admission, the patient's blood pressure was 107/83 $\mathrm{mmHg}$ and his heart rate was 100 beats/min. His oxygen saturation level was $90 \%$ (room air). The results of the arterial blood gas analysis on $2 \mathrm{~L}$ of $\mathrm{O}_{2}$ indicated hypoxemia $\left(\mathrm{PaO}_{2} 69.8 \mathrm{mmHg}\right.$ and $\left.\mathrm{PaCO}_{2} 30.4 \mathrm{mmHg}\right)$. He had a heavy, dry cough and found it difficult to breathe. He also had marked limitations of physical activity, and could not complete the 6-minute walk test.

Chest radiography showed that the patient's lung field was clear. An electrocardiogram showed a right axis deviation and pulmonary $\mathrm{P}$ wave in II, III, and aVF. The labora-

\footnotetext{
${ }^{1}$ Department of Cardiovascular Medicine and Hypertension, Graduate School of Medical and Dental Sciences, Kagoshima University, Japan and ${ }^{2}$ Department of Human Pathology, Field of Oncology, Graduate School of Medical and Dental Sciences, Kagoshima University, Japan Received for publication December 25, 2013; Accepted for publication April 29, 2014 Correspondence to Dr. Kayoko Kubota, kubota@m.kufm.kagoshima-u.ac.jp
} 

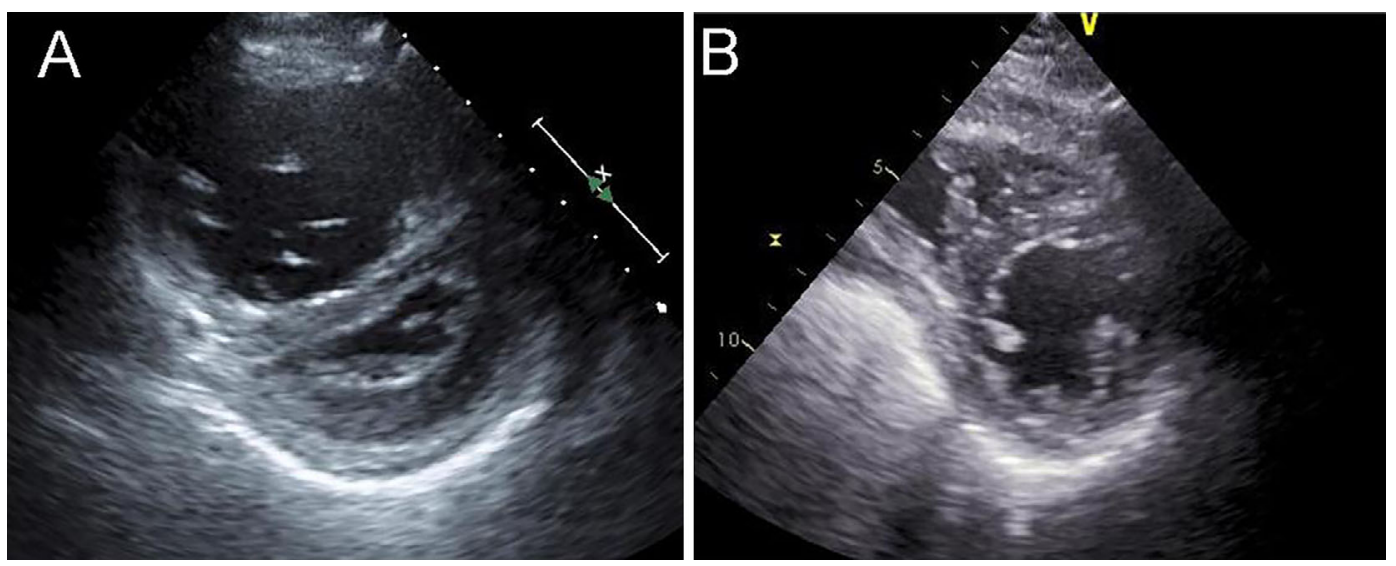

Figure 1. Pulmonary hypertension and treatment. (A) Echocardiogram on admission of the short axis views at the diastolic phase shows a dilated right ventricle with a compressed left ventricle. (B) Echocardiogram three months after combination therapy shows substantial recovery.

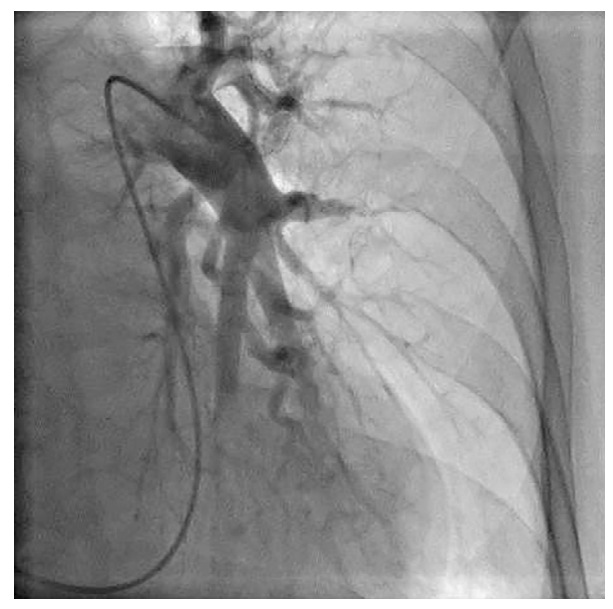

Figure 2. A pulmonary angiogram depicts delayed pulmonary blood flow. The pulmonary angiogram does not show abrupt vascular narrowing or an intravascular web.

tory data were as follows: white blood cells, 6,920 cells/ $\mathrm{mm}^{3}$, C-reactive protein levels, $1.3 \mathrm{mg} / \mathrm{dL}$; and brain natriuretic peptide levels, $746 \mathrm{pg} / \mathrm{mL}$. The results from the blood analysis did not suggest any collagen diseases leading to pulmonary hypertension. Positron emission tomography and computed tomography (PET-CT) showed that there was no recurrence of the rectal cancer, but small lung metastases were found. A dilated right ventricle was observed by the echocardiogram, and the estimated right ventricular systolic pressure was $85.7 \mathrm{mmHg}$ (Fig. 1A). A mosaic pattern was observed by chest CT, but pulmonary thromboembolism was not detected. Lung perfusion scintigraphy showed subsegmental defects. These findings suggested chronic thromboembolic pulmonary hypertension; however, the symptoms of chronic thromboembolic pulmonary hypertension gradually progress, while the symptoms of this patient dramatically worsened.

After referring to the past history of colorectal cancer in this patient, PTTM was suspected. Pulmonary hypertension was confirmed by a right heart catheterization with a normal pulmonary angiogram (Fig. 2). His pulmonary arterial pressure was $77 / 31(48) \mathrm{mmHg}$ and the cardiac index was 1.82 $\mathrm{L} / \mathrm{min} / \mathrm{m}^{2}$. The patient's blood was then aspirated from the pulmonary artery catheter in the wedge position. Tumor cells were detected in the blood sample through immunohistochemistry. Immunohistochemical staining was performed via the immunoperoxidase method. Briefly, following incubation with each of the primary antibodies in phosphate buffered saline $(\mathrm{pH} \mathrm{7.4,} \mathrm{containing} 1 \%$ bovine serum albumin), sections were stained on a Benchmark XT automated slide stainer using a diaminobenzidine detection kit (ultraView DAB, Ventana Medical Systems, Tucson, USA) (Fig. 3). Due to the very low yield of cells from the pulmonary capillary wedge aspiration, the histology results were not used. The patient was then diagnosed with PTTM. Bosentan and tadalafil were prescribed to decrease the pulmonary hypertension, and furosemide and spironolactone were administered in order to treat his heart failure. To eradicate the pulmonary tumor cells, imatinib $(50 \mathrm{mg} /$ day $)$ was prescribed and gradually increased to $200 \mathrm{mg} /$ day. Bevacizumab was administered intravenously every 2 weeks at a dose of $5 \mathrm{mg} / \mathrm{kg}$, and $100 \mathrm{mg} /$ day S-1 (tegafur, gimeracil, and oteracil potassium) was prescribed for four weeks and alternately discontinued for two weeks.

On admission, the patient was unable to walk; however, after he began to take the medications, he could walk to the bathroom by himself. Additionally, his dry cough rapidly improved after the initiation of these medications. We did not conduct anticoagulation therapy because one possible side effect of bevacizumab treatment includes bleeding. No other side effects were apparent.

The patient's serum brain natriuretic peptide level gradually decreased from 746 to $23 \mathrm{pg} / \mathrm{dL}$. An echocardiogram showed improvement in the estimated right ventricular systolic pressure from 85.7 to $55.7 \mathrm{mmHg}$ (Fig. 1B). Right heart catheterization was performed 12 weeks after the initiation of the medications. The patient's pulmonary arterial pressure was $69 / 17$ (35) $\mathrm{mmHg}$ and the cardiac index was 

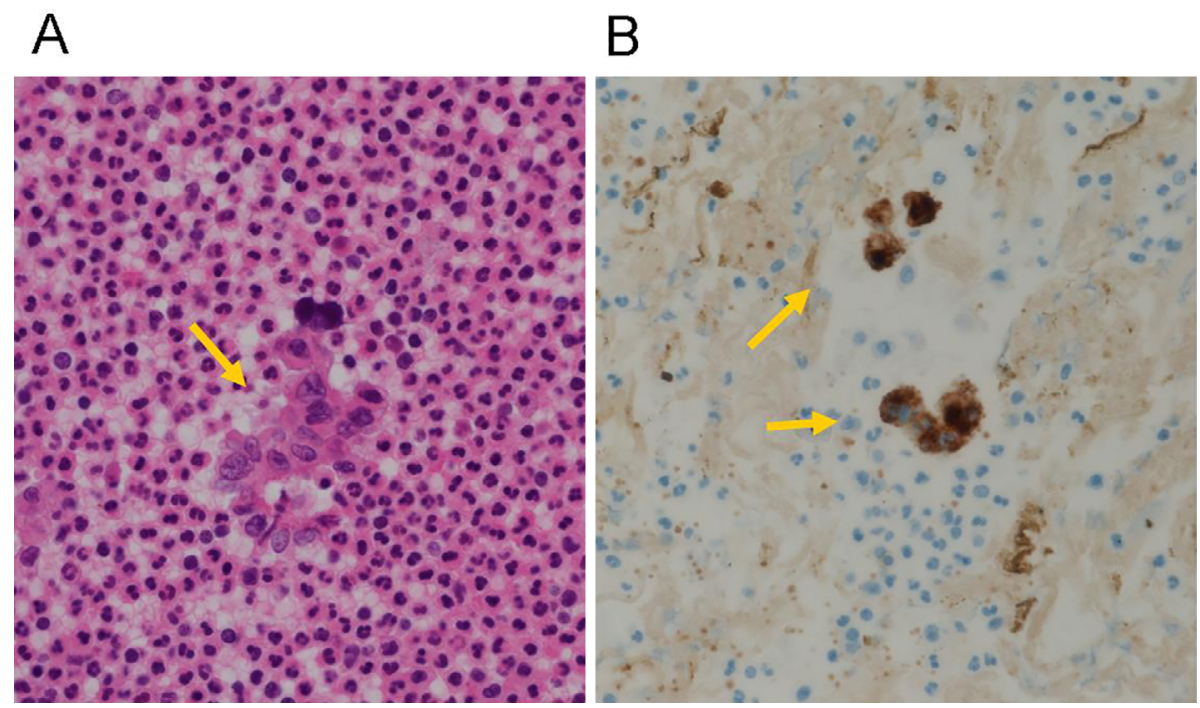

Figure 3. Immunohistochemical identification of the tumor cells in the pulmonary arterial blood. (A) Tumor cells observed in the pulmonary arterial blood (arrow) (Hematoxylin and Eosin staining, 400x). (B) Immunohistochemical findings depict small clusters of tumor cells (arrows) (MUC4/1G8, 400x).

\section{$4.64 \mathrm{~L} / \mathrm{min} / \mathrm{m}^{2}$.}

Although the administration of $5 \mathrm{~L}$ of oxygen was required, the patient was discharged after three months of hospitalized treatment. He did not experience worsening of his pulmonary hypertension. However, he died from respiratory failure due to an influenza infection 12 months later.

\section{Discussion}

The mechanisms of action of PTTM are thought to occur through tumor cell occlusion of the small arteries and arterioles, the activation of coagulation systems, and the release of inflammatory mediators and growth factors (3). Based on the histological findings, PTTM is often associated with adenocarcinomas, especially the poorly differentiated type. The most common origin of PTTM is in the stomach, and other origins include the lung, breast, and colon (1).

Because of the extremely rapid progression of PTTM, making an early diagnosis and administering therapeutic intervention are important. Several cases have been reported where combination chemotherapy with S-1 and cisplatin achieved some therapeutic effects $(4,5)$. Pulmonary microvascular cytology using a wedged pulmonary artery catheter has been suggested as a good tool for use in the antemortem diagnosis of PTTM $(6,7)$. Our patient was also diagnosed using this method.

In previous immunohistochemical studies, VEGF, tissue factor, and PDGF were found to be important molecules in the pathogenesis of PTTM (3, 8-10). PDGF and PDGF receptors are expressed in carcinoma cells and endothelial cells (11).

Imatinib is a PDGF receptor antagonist, which subsequently blocks many downstream intracellular signaling pathways. Ogawa et al. reported that imatinib treatment was effective in patients with PTTM (12). They described that the PDGF expression levels may have been modified by imatinib therapy. In our case, the patient's serum PDGF levels decreased after combination therapy (from 3,710 to $1,380 \mathrm{pg} / \mathrm{mL}$ ).

VEGF is an endothelial, cell-specific, angiogenetic mitogen implicated in several physiological and pathological processes that require proliferation of endothelial cells. VEGF is also involved in pulmonary hypertension (13). VEGF expression by tumor cells has recently been confirmed in many PTTM cases $(3,8-10)$. These reports suggest that VEGF may be involved in the pathogenesis of PTTM. The molecular mechanisms underlying the development of PTTM remain unclear. In our case, PDGF and VEGF could not be detected in the tumor cells due to damage to the cells caused by the pulmonary capillary wedge aspiration. Bevacizumab is a VEGF receptor inhibitor. Although bevacizumab was discontinued for treatment of the colorectal cancer due to suspected side effects, the patient's symptoms rapidly worsened after discontinuation. We suspected that bevacizumab would be effective for PTTM, therefore, we readministered it, and the VEGF levels decreased after the therapy (from 165 to $76.5 \mathrm{pg} / \mathrm{mL}$ ). In 30 autopsy cases of PTTM, the immunohistochemical positivity for VEGF and tissue factor was high, whereas that for PDGF was relatively low, suggesting that VEGF and tissue factor may play important roles in the pathogenesis of PTTM (10). There are few reports of patients who were diagnosed with PTTM where the outcome improved. The reasons for our successful treatment may be due to the immediate diagnosis of PTTM with pulmonary wedge aspiration and the combination therapy of imatinib, bevacizumab, S-1, and cisplatin. Although the antemortem diagnosis of PTTM is extremely difficult, based on the findings in our case, a 


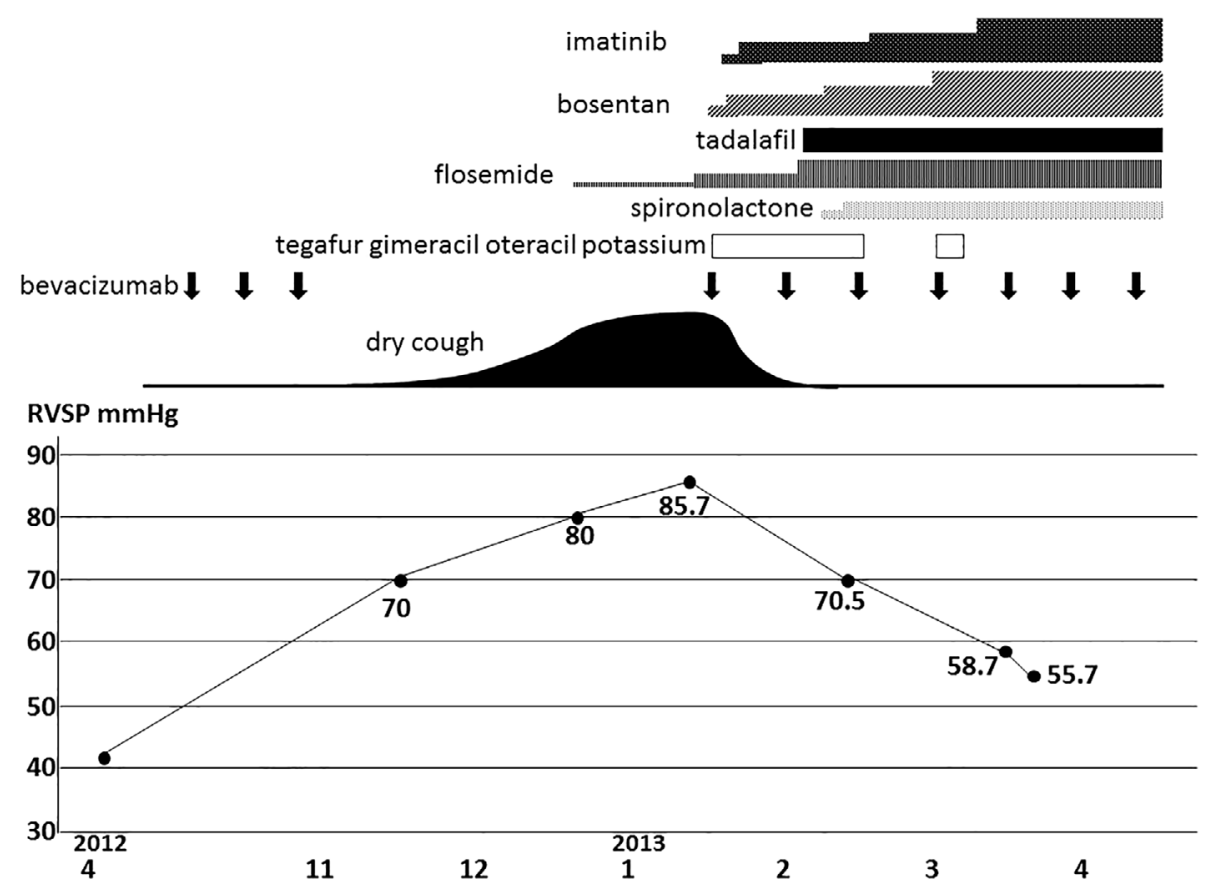

Figure 4. Time course of the treatment. Right ventricular systolic pressure (RVSP) is shown. The patient's dry cough rapidly improved after the initiation of bevacizumab and imatinib.

severe, dry cough may be a useful criterion for the antemortem diagnosis. This is based on the notion that some cytokines may induce airway hyperresponsiveness in patients with PTTM, thereby leading to the onset of a dry cough.

There are insufficient data on the effective strategies for PTTM treatment. PTTM is an important differential diagnosis for patients with rapidly worsening pulmonary hypertension with a severely dry cough, with or without a history of cancer. PTTM is found in $3.3 \%$ of the autopsies of patients with malignant tumors (1). An early diagnosis may therefore help to improve the outcome of patients with PTTM.

As observed in our case, the prognosis of PTTM may be improved by combination therapy. The most effective agent in this combination therapy is unclear. The patient's dry cough improved rapidly within a few days of starting imatinib and bevacizumab.

We therefore considered that molecular-target drugs were effective against PTTM through his clinical course (Fig. 4). Although the mechanism through which our patient's cough immediately improved by imatinib and bevacizumab treatment is still unclear, we believe that the effects of these molecular-target drugs may appear relatively quickly because his symptoms worsened within one month after stopping bevacizumab in the previous hospital. Conversely, careful and sufficient discussion regarding bevacizumab treatment might be necessary because pulmonary hypertension is reported as one of the possible side effects of bevacizumab (14). Comprehensive studies are needed to elucidate the mechanism by which molecular-target drugs exert their effects during PTTM.

The authors state that they have no Conflict of Interest (COI).

\section{References}

1. von Herbay A, Illes A, Waldherr R, Otto HF. Pulmonary tumor thrombotic microangiopathy with pulmonary hypertension. Cancer 66: 587-592, 1990.

2. Gavin MC, Morse D, Partridge AH, Levy BD, Loscalzo J. Clinical problem-solving. Breathless. N Engl J Med 366: 75-81, 2012.

3. Chinen K, Kazumoto T, Ohkura Y, Matsubara O, Tsushiya E. Pulmonary tumor thrombotic microangiopathy caused by a gastric carcinoma expressing vascular endothelial growth factor and tissue factor. Pathol Int 55: 27-31, 2005.

4. Miyano S, Izumi S, Takeda Y, et al. Pulmonary tumor thrombotic microangiopathy. J Clin Oncol 25: 597-599, 2007.

5. Kayatani H, Matsuo K, Ueda Y, et al. Pulmonary tumor thrombotic microangiopathy diagnosed antemortem and treated with combination chemotherapy. Intern Med 51: 2767-2770, 2012.

6. Masson RG, Ruggieri J. Pulmonary microvascular cytology. A new diagnosis application of the pulmonary artery catheter. Chest 88: 908-914, 1985.

7. Babar SI, Sobonya RE, Snyder LS. Pulmonary microvascular cytology for the diagnosis of pulmonary tumor embolism. West $\mathbf{J}$ Med 168: 47-50, 1998.

8. Okubo Y, Wakayama M, Kitahara K, et al. Pulmonary tumor thrombotic microangiopathy induced by gastric carcinoma: morphometric and immunohistochemical analysis of six autopsy cases. Diagn Pathol 6: 27, 2011.

9. Chinen K, Tokuda Y, Fujiwara M, Fujioka Y. Pulmonary tumor thrombotic microangiopathy in patients with gastric carcinoma: an analysis of 6 autopsy cases and review of the literature. Pathol Res Pract 206: 682-689, 2010.

10. Uraga H, Fujii T, Kurosaki A, et al. Pulmonary tumor thrombotic microangiopathy: a clinical analysis of 30 autopsy cases. Intern Med 52: 1317-1323, 2013.

11. Yokomine T, Hirakawa H, Ozawa E, Shibata K, Nakayama T. Pulmonary thrombotic microangiopathy caused by gastric carcinoma. J Clin Pathol 63: 367-369, 2010.

12. Ogawa A, Yamadori I, Matsubara O, Matsubara H. Pulmonary tu- 
mor thrombotic microangiopathy with circulatory failure treated with imatinib. Intern Med 52: 1927-1930, 2013.

13. Geiger R, Berger RM, Hess J, Bogers AJ, Sharma HS, Mooi WJ Enhanced expression of vascular endothelial growth factor in pulmonary plexogenic arteriopathy due to congenital heart disease. J Pathol 191: 202-207, 2000.
14. Garcia AA, Hirte H, Fleming G, et al. Phase II clinical trial of bevacizumab and low-dose metronomic oral cyclophosphamide in recurrent ovarian cancer: a trial of the California, Chicago, and Princess Margaret Hospital phase II consortia. J Clin Oncol 26: 76-82, 2008.

(C) 2014 The Japanese Society of Internal Medicine http://www.naika.or.jp/imonline/index.html 